\title{
MONOAMINE OXIDASE INHIBITION IMPROVES VASCULAR FUNCTION IN MAMMARY ARTERIES FROM NON-DIABETIC AND DIABETIC PATIENTS WITH CORONARY HEART DISEASE
}

\begin{tabular}{|c|c|}
\hline Journal: & Canadian Journal of Physiology and Pharmacology \\
\hline Manuscript ID & cjpp-2015-0580.R1 \\
\hline Manuscript Type: & Article \\
\hline Date Submitted by the Author: & 27-Jan-2016 \\
\hline Complete List of Authors: & $\begin{array}{l}\text { Lighezan, Rodica; "Victor Babes" University of Medicine and Pharmacy, } \\
\text { Department of Parasitology; "Victor Babes" University of Medicine and } \\
\text { Pharmacy, Center for Translational Research and Systems Medicine } \\
\text { Sturza, Adrian; University of Medicine and Pharmacy of Timisoara, } \\
\text { Department of Pathophysiology; "Victor Babes" University of Medicine and } \\
\text { Pharmacy, Center for Translational Research and Systems Medicine } \\
\text { Duicu, Oana; "Victor Babes" University of Medicine and Pharmacy, } \\
\text { Department of Pathophysiology; "Victor Babes" University of Medicine and } \\
\text { Pharmacy, Center for Translational Research and Systems Medicine } \\
\text { Ceausu, Raluca; "Victor Babes" University of Medicine and Pharmacy, } \\
\text { Department of Histology } \\
\text { Vaduva, Adrian; University of Medicine and Pharmacy of Timisoara, } \\
\text { Morphopathology } \\
\text { Gaspar, Marian; "Victor Babes" University of Medicine and Pharmacy, } \\
\text { Department of Cardiovascular Surgery } \\
\text { Feier, Horea; "Victor Babes" University of Medicine and Pharmacy, } \\
\text { Department of Cardiovascular Surgery } \\
\text { Vaida, Monica ; "Victor Babes" University of Medicine and Pharmacy, } \\
\text { Department of Anatomy } \\
\text { Ivan, Viviana; "Victor Babes" University of Medicine and Pharmacy, } \\
\text { Department of Cardiology - 2nd Cardiology Clinic } \\
\text { Lighezan, Daniel; "Victor Babes" University of Medicine and Pharmacy, } \\
\text { Department of Internal Medicine; "Victor Babes" University of Medicine and } \\
\text { Pharmacy, Center for Translational Research and Systems Medicine } \\
\text { Muntean, Danina; "Victor Babes" University of Medicine and Pharmacy, } \\
\text { Timisoara, RO, Department of Pathophysiology; "Victor Babes" University } \\
\text { of Medicine and Pharmacy, Center for Translational Research and Systems } \\
\text { Medicine } \\
\text { Mornoş, Cristian; "Victor Babes" University of Medicine and Pharmacy, } \\
\text { Department of Cardiology - 1st Cardiology Clinic }\end{array}$ \\
\hline Keyword: & $\begin{array}{l}\text { : monoamine oxidases, coronary artery disease, diabetes mellitus, } \\
\text { endothelial dysfunction, MAO inhibitors }\end{array}$ \\
\hline
\end{tabular}


SCHOLARONE $^{\text {m }}$
Manuscripts

https://mc06.manuscriptcentral.com/cjpp-pubs 


\section{MONOAMINE OXIDASE INHIBITION IMPROVES VASCULAR FUNCTION IN MAMMARY ARTERIES FROM NON-DIABETIC AND DIABETIC PATIENTS WITH CORONARY HEART DISEASE}

Rodica Lighezan ${ }^{1 *}$, Adrian Sturza ${ }^{2, \#, *}$, Oana M. Duicu ${ }^{2, \#}$, Raluca A. Ceausu ${ }^{3}$, Adrian Vaduva $^{4}$, Marian Gaspar ${ }^{5}$, Horea Feier ${ }^{5}$, Monica Vaida ${ }^{6}$, Viviana Ivan $^{7}$, Daniel Lighezan ${ }^{8, \#}$, Danina M. Muntean ${ }^{2, \#}$, Cristian Mornos ${ }^{9}$

${ }^{1}$ Department of Parasitology, ${ }^{2}$ Department of Pathophysiology, ${ }^{3}$ Department of Histology,

${ }^{4}$ Department of Morphopathology, ${ }^{5}$ Department of Cardiovascular Surgery, ${ }^{6}$ Department of Anatomy, ${ }^{7}$ Department of Cardiology-2 $2^{\text {nd }}$ Cardiology Clinic, ${ }^{8}$ Department of Internal Medicine-I, ${ }^{9}$ Department of Cardiology- ${ }^{\text {st }}$ Cardiology Clinic, "Victor Babeș" University of Medicine and Pharmacy, Timișoara, Romania

\#Center for Translational Research and Systems Medicine, "Victor Babeș" University of Medicine and Pharmacy, Timişoara, Romania

Corresponding author: Danina M. Muntean

Department of Pathophysiology,

"Victor Babeș" University of Medicine and Pharmacy of Timişoara

2, Eftimie Murgu Sq., 300041 Timisoara, RO

Tel/Fax: +40-256-493085

E-mail: daninamuntean@,umft.ro

*These authors contributed equally to this work. 


\begin{abstract}
Monoamine oxidases (MAOs) are mitochondrial enzymes with 2 isoforms that have emerged as important contributors to cardiovascular oxidative stress via the constant generation of hydrogen peroxide. The present study was purported to assess whether MAO-derived $\mathrm{H}_{2} \mathrm{O}_{2}$ contributes to the endothelial dysfunction in mammary arteries harvested from coronary heart disease patients with/without diabetes mellitus subjected to coronary artery bypass grafting. To this aim the effects of MAO inhibition on vascular contractility to phenylephrine and endothelial-dependent relaxation (EDR) in response to acetylcholine were studied in vascular segments. Clorgyline (irreversible MAO A inhibitor), selegiline (irreversible MAO B inhibitor), and moclobemide (reversible MAO A inhibitor) were applied in the organ bath $(10 \mu \mathrm{mol} / \mathrm{L})$. MAO expression was assessed by immunohistochemistry. We found a constant impairment of EDR that has been significantly attenuated in the presence of the MAO A and $\mathrm{B}$ inhibitors in both groups of coronary heart disease patients. MAO B was the dominant isoform in all human diseased vessels. In conclusion, in vitro inhibition of MAO significantly improved endothelium-dependent relaxation in human mammary arteries, regardless the presence of diabetes. These data suggest that MAO inhibitors might be useful in restoring endothelial response in clinical conditions associated with increased oxidative stress, such as coronary artery disease and diabetes.
\end{abstract}

Key words: monoamine oxidases, coronary artery disease, diabetes mellitus, endothelial dysfunction, MAO inhibitors

\title{
Introduction
}

Coronary heart disease (CHD) is currently the leading cause of morbidity due to heart failure and its evolution is aggravated by the presence of diabetes mellitus (DM) whose prevalence is increasing at an alarming rate worldwide. Nowadays, it is widely accepted that 
excessive generation of reactive oxygen species (ROS) plays a central role in the pathogenesis of cardiovascular diseases, contributing to both endothelial and mitochondrial dysfunction that, in turn, accelerate their evolution towards complications.

Mitochondria have emerged as the major sources of oxidative stress in the cardiovascular system; in particular, complexes I and III of the respiratory chain at the internal mitochondrial membrane are primarily responsible for occasional generation of superoxide anion (Fridovich 1995; St-Pierre et al. 2002). However, another mitochondrial enzyme responsible for the obligatory hydrogen peroxide $\left(\mathrm{H}_{2} \mathrm{O}_{2}\right)$ production at the outer mitochondrial membrane of cardiomyocytes has attracted the interest of the researchers (recently reviewed in (Kaludercic et al. 2014). Monoamine oxidase (MAO) with 2 isoforms, $\mathrm{A}$ and $\mathrm{B}$, are flavoproteins that catalyze the electron transfer from amines (exogenous and endogenous) and neurotransmitters to $\mathrm{O}_{2}$, with the constant generation of $\mathrm{H}_{2} \mathrm{O}_{2}$ as by-product (Di Lisa et al. 2009).

A large body of experimental evidence showed that under physiological conditions the amount of $\mathrm{H}_{2} \mathrm{O}_{2}$ generated via $\mathrm{MAO}$ is reduced but in cardiac pathologies (such as myocardial ischemia/reperfusion and heart failure) the increased activity of the MAO-A isoform becomed deleterious (Bianchi et al. 2005a; Kaludercic et al. 2011; Kaludercic et al. 2010). Accordingly, in the rodent heart, MAO-A activation contributes to the ischemia/reperfusion injury in the in vivo model of regional ischemia (Bianchi et al. 2005a,b; Carpi et al. 2009) and to the maladaptive ventricular remodeling in the transverse aortic constriction-induced model of heart failure, respectively (Kaludercic et al. 2010; Villeneuve et al. 2013). Furthermore, an elegant study has unequivocally proven the role of MAO-B activation in triggering mitochondrial dysfunction besides the cardiac structural and functional alterations in mice with experimentally induced heart failure (Kaludercic et al. 2014). 
Recently, we described the role of MAOs as mediators of endothelial dysfunction and the beneficial effect of MAO inhibition in murine diseased vessels (Sturza et al. 2013), and in experimental diabetes mellitus, respectively (Duicu et al. 2015; Sturza et al. 2015).

The present study aims at assessing the role of MAOs as contributors to the endothelial dysfunction in mammary arteries from CHD patients with and without DM subjected to coronary artery by-pass grafting.

\section{Materials and methods}

Samples of internal mammary arteries were obtained from patients with preserved ejection fraction that underwent coronary artey bypass grafting. Patients were randomized in 2 groups: (1) patients with coronary heart disease (CHD) without DM (CHD group), and (2) coronary artery disease patients with documented DM (CHD-DM group).

The University Committee for Ethics Research approved the study protocol and the informed consent was obtained from all patients prior to surgery, according to the World Medical Association Declaration of Helsinki.

Demographic, clinical data and preoperative medication for both groups are presented in Table 1. The characteristics of the patients included in the study are represented in Table 1.

\section{Preparation of the mammary arteries and isometric tension measurements. After} harvesting the left internal mammary artery using the skeletonized technique, the distal end $(1-2 \mathrm{~cm})$ of the artery was divided, placed in cold Hanks's solution and immediately transferred to the laboratory. The diameter of the samples varied according to the specific anatomy of the patient, but was generally between 1.25-2 mm. Organ bath experiments were performed in human internal mammary artery rings in the presence of diclofenac $(10 \mu \mathrm{mol} / \mathrm{L})$. The concentration of phenylephrine was adjusted to obtain a preconstriction level of $80 \%$ of 
the contraction elicited by $\mathrm{KCl}(80 \mathrm{mmol} / \mathrm{L})$. Endothelium-dependent relaxation to cumulative concentrations of acetylcholine (Ach) was recorded in the presence or absence of the following MAO inhibitors $(10 \mu \mathrm{mol} / \mathrm{L})$ : clorgyline - the irreversible MAO-A inhibitor, moclobemide - the reversible MAO-A inhibitor and selegiline - the irreversible MAO-A inhibitor (incubation time, $30 \mathrm{~min}$ ). In separate experiments, endothelium was removed by a short treatment with CHAPS ( $5 \mathrm{mg} / \mathrm{mL}$ dissolved in glucose solution $50 \mathrm{~g} / \mathrm{L}, 40$ seconds).

Immunohistochemistry. Internal mamary arteries samples from patients admitted for the revascularization procedure were primary processed for morphological diagnosis and immunohistochemistry. Immediate after prelevation, specimens were fixed by immersion in $4 \%$ buffered formalin, embedded in paraffin and sectioned at $3-5 \mu \mathrm{m}$. The slides were then dewaxed and rehydrated and either stained with the usual Hematoxylin-Eosin (HE) or pretreated for immunohistochemistry. For immunostaining we used the following antibodies: Monoamine Oxidase A and Monoamine Oxidase B (Novus Biologicals, USA). We performed a heat-induced epitope retrieval, for 20 minutes, with Novocastra Bond Epitope Retrieval Solution 1, a citrate based $\mathrm{pH} 6.0$ solution, ready-to-use, (Leica Biosystems, UK). Endogenous peroxidase activity was inhibited by a 5 min preincubation in a solution containing 3\% hydrogen peroxide. This step was followed by the incubation with the primary antibodies: Monoamine Oxidase A (mouse monoclonal anti-human, 1D6, dilution 1:300, Novus Biologicals, USA), and Monoamine Oxidase B (rabbit polyclonal anti-human, dilution 1:50, Novus Biologicals, USA) for 30 minutes. The Bond Polymer Refine Detection System was applied for visualisation, and 3,3 diamino-benzidine dihydrochloride was applied as chromogen for 10 minutes. The nuclei were counterstained with Mayer's hematoxylin for 5 minutes. The entire immunohistochemical procedure was performed with a Leica Bond-Max autostainer (Leica Biosystems, Newcastle uponTyne, UK). After staining, the slides were 
dehydrated and mounted with Canada balsam. Image acquisition and analysis were performed using a Nikon Eclipse E 600 microscope. Immunohistochemical expression of intracytoplasmatic MAO isoenzymes was scored using a semi-quantitative intensity scale: absent, weak, moderate and strong intensity.

\section{Statistics}

Data analysis of the dose-effect response curves was performed using the ANOVA F-test (comparisons of bottom and top values, EC50 and the Hill slope). Values of $p<0.05$ were considered statistically significant.

\section{Results}

\section{Ex Vivo Inhibition of MAOs Reduces Contractility And Improves Relaxation Of Internal Mamary Arteries}

MAOs have emerged as novel mediatos of endothelial dysfunction in animal models. In order to confirm this hypothesis in humans, the effect of MAO inhibition on endothelial function in human mammary arteries was assessed. Firstly, the contractile effect to cumulative doses of phenylephrine (Phe) was tested. In all patients we report a significant rightward shift of the concentration-response curve of Phe in the mammary arteries treated with MAO inhibitors (MAOI) as compared to the none-treated vessels (Fig. 1 A, C). Subsequently, we measured the endothelium-dependent relaxation in response to cumulative doses of acetylcholine (ACh) in the presence $v s$. the absence of three MAOI: clorgyline, moclobemide and selegiline (10 $\mu \mathrm{mol} / \mathrm{L})$. Similarly, in both groups, all MAO inhibitors significantly improved the relaxation response to ACh (Fig. 1 B, D). No significant difference was found between diabetic and nondiabetic patients with respect to the beneficial effects of MAOI on endothelium-dependent relaxation (data not shown). 


\section{MAO Is Expressed In All Layers of Human Mammary Arteries}

\section{Distribution and cellular localization}

Hematoxylin-eosin staining of the human mammary arteries did not revealed any morphological differences between the CHD and CHD-DM patients (Fig.2). Subsequently, we evaluated the immunohistochemical expression of MAO-A and MAO-B in mammary arteries of CHD patients, with and without diabetes and showed that both isoforms of MAO (A and B) were present in the cytoplasm of various cells within all the three layers of the vascular wall; accordingly, MAO isoenzymes were expressed in endothelial cells and fibroblasts of the intima, in smooth muscle cells of the media (Fig.3 b,e; Fig.4 b,e) and in fibroblasts and adipocytes of the adventitia (Fig.3 c,f; Fig.4 c,f).

\section{Intensity}

There was a moderate MAO-A expression and a strong MAO-B expression in the vascular wall of both patients groups as depicted in Fig. 3 and 4. However, no significant difference was found in either isoenzyme expression between the diabetic and the non-diabetic group.

\section{The Presence of An Intact Endothelium Is Crucial For The Vascular Effects of MAOI}

Ex vivo treatment with MAOI significantly reduced contractility and improved the relaxation response in human mammary arteries in all CHD patients with and without diabetes. However, endothelial denudation with CHAPS completely abolished the effects of the pharmacological inhibitors on vascular reactiviy (Fig. 5). 


\section{Discussion}

In the present study we focused on the role of MAOs in the pathogenesis of endothelial dysfunction in patients with CHD with and without diabetes. We detected MAO expression in internal mammary arteries, the enzyme being distributed throughout the entire structure of the vascular wall: the endothelial and muscular layers, and adventitia (with nerves, perivascular adipose and conjunctive tissue). In both groups MAO-B was the dominant isoform.

In a previous study we reported the upregulation of both MAO isoforms in aortic rings harvested from rats with streptozotocin-induced diabetes as compared to the control animals (Sturza et al. 2015). At variance, in the present study there were no differences in MAO expression between the diabetic and non-diabetic patients, but one must bear in mind that they were all diagnosed with coronary artery disease. As mentioned in the Material and Methods section, we selected patients with preserved left ventricular ejection fraction in order to prevent a confounding factor, since it has been reported that systolic heart failure is a leading cause of MAO overexpression in the rodent heart (Kaludercic et al. 2011). However, several factors could interfere with MAO expression in the present study. Firstly, it has to be mentioned that all recruited patients were also treated hypertensives. Hypertension is a risk factor clearly involved in the maintenance of chronic inflammation and progression of atherosclerotic lesions (Rosendorff et al. 2015). In experimental settings, we have previously reported that acute administration of lipopolysaccharide (with subsequent inflammation) or chronic delivery of angiotensin II (with subsequent hypertension) lead to overexpression of both MAO isoforms, increase in ROS production and impairment of vascular relaxation (Sturza et al. 2013). Second, medication can also modify the enzyme expression. In particular, it has been reported that treatment with angiotensin II converting enzyme inhibitors suppressed MAO activity (Raasch et al. 2002). Of note, in our study both diabetic and non- 
diabetic patients were treated with this class of drugs. Interestingly, it has been also reported that pioglitazone, an anti-diabetic thiazolidinedione, acts as a specific and reversible inhibitor of human MAO-B (Binda et al. 2011).

The other important finding of our study is that blockade of endogenous MAO activity partially reduced contractility and improved the endothelium-dependent relaxation in vascular rings harvested from all CHD patients, regardless the presence or the absence of diabetes. This finding strongly suggests that: i) MAO-related ROS production contributes to the prooxidative status underlying endothelial dysfunction in CHD patients and ii) MAOinhibition is able to partially reverse the functional impairment. The observations are in agreement with our previous findings in experimental models of diabetes: ex vivo incubation of the aortic rings with the same MAO inhibitors attenuated the endothelial dysfunction in aortic rings harvested from rats with streptozotocin induced diabetes (the classic model of type 1 DM) (Sturza et al. 2015) as well as in Zucker diabetic fatty rats (a genetic model of type 2 DM) (Sturza et al. 2014).

Last but not least, the most important finding of this study from pathophysiological point of view is related to the lack of beneficial effects of MAOI in the absence of a functional endothelium. Indeed, in denuded mammary arteries no alleviation of endothelial dysfunction was further obtained, in the presence of all MAO inhibitors. We did not address the signal transduction sequence responsible for the beneficial vascular effects of MAOI and we acknowledge this study limitation. However, we speculate that a decrease in nitric oxide (NO) availability is the underlying mechanism. Indeed, we have previously reported in HUVECs that MAO-A directly limits the accumulation of cGMP and thus, NO formation (Sturza et al. 2013). However, other pleiotropic effects of MAOI cannot be excluded.

In particular, selegiline, commonly used in the treatment of Parkinson and Alzheimer diseases (Thomas 2000), has been reported to increase the level of nitric oxide in the brain 
and in cerebral blood vessels, regardless of the MAO-B inhibition (Thomas et al. 1998). Also, the inhibitor protects the endothelium against the toxic effect of amyloid, increase the level of superoxide dismutase in the brain, prevent apoptosis mediated by peroxynitrite and protect dopaminergic neurons from the toxicity mediated by glutamate or induced by the depletion of glutathione (Ebadi et al. 2006; Thomas et al. 1998).

MAO inhibitors are currently used in psychiatry and neurology for the treatment of anxiety and depressive disorders, Parkinson's and Alzheimer's diseases, respectively. Therefore, no major problem should exist for the clinical assessment of their potential beneficial cardiovascular effects. Of note, MAO inhibition was largely used in the late 60 s, mostly in the ex-Soviet Union, in the treatment of angina pectoris and hypertension (Griffith 1960; Kushelevskii and Kokosov 1967; Simon 1970).

Since MAOs are the major enzymes responsible for catecholamine degradation, it will be predicted in clinical setting to favor the global accumulation of these vasoactive compounds with an increased risk for the occurrence of hypertensive crises (after ingestion of food, e.g. fermented cheese rich in tyramine, the norepinephrine precursor, concomitant with MAO inhibitors will elicit vasoconstriction - the well-known 'cheese-effect') as a direct consequence of the irreversible MAO inhibition (Finberg and Gillman 2011). However, whether this holds also true for the large scale-used novel reversible MAO inhibitors it is not completely clear.

Cardiovascular therapy is a rapidly changing field that witnessed important paradigm shifts, one of the most important being the use of beta blockers in heart failure, once regarded as counterintuitive. Therefore, in light of the exciting findings pointing to the role of MAOrelated oxidative stress in cardiovascular pathology, the reversible MAO inhibitors represent a promising class for the therapeutic armamentarium of both heart and brain diseases. 


\section{Conclusions}

In conclusion, monoamine oxidases are expressed in human mammary arteries but it is the MAO-B isoform that is mainly induced in coronary heart disease patients with preserved ejection fraction. In vitro inhibition of MAOs significantly improved endothelium-dependent relaxation in human mammary arteries, regardless the presence of diabetes. These data suggest that MAO inhibitors might be useful in restoring endothelial response in clinical conditions associated with increased oxidative stress and vascular dysfunction such as coronary artery disease and diabetes.

\section{Acknowledgments}

Research supported by the university grant PIII-C1-PCFI-2014/2015-04.

\section{Conflicts of interest}

The authors confirm that this article content has no conflicts of interest.

\section{References}

Bianchi, P., Kunduzova, O., Masini, E., Cambon, C., Bani, D., Raimondi, L., Seguelas, M.H., Nistri, S., Colucci, W., Leducq, N., and Parini, A. 2005a. Oxidative stress by monoamine oxidase mediates receptor-independent cardiomyocyte apoptosis by serotonin and postischemic myocardial injury. Circulation, 112(21): 3297-3305. doi: 10.1161/circulationaha.104.528133. 
Bianchi, P., Pimentel, D.R., Murphy, M.P., Colucci, W.S., and Parini, A. 2005b. A new hypertrophic mechanism of serotonin in cardiac myocytes: receptor-independent ROS generation. FASEB J. 19(6): 641-643. doi: 10.1096/fj.04-2518fje.

Binda, C., Aldeco, M., Geldenhuys, W.J., Tortorici, M., Mattevi, A., and Edmondson, D.E. 2011. Molecular Insights into Human Monoamine Oxidase B Inhibition by the Glitazone Anti-Diabetes Drugs. ACS Med. Chem. Lett. 3(1): 39-42. doi: 10.1021/ml200196p.

Carpi, A., Menabo, R., Kaludercic, N., Pelicci, P., Di Lisa, F., and Giorgio, M. 2009. The cardioprotective effects elicited by $\mathrm{p} 66(\mathrm{Shc})$ ablation demonstrate the crucial role of mitochondrial ROS formation in ischemia/reperfusion injury. Biochim. Biophys. Acta, 1787(7): 774-780. doi: 10.1016/j.bbabio.2009.04.001.

Di Lisa, F., Kaludercic, N., Carpi, A., Menabo, R., and Giorgio, M. 2009. Mitochondrial pathways for ROS formation and myocardial injury: the relevance of p66(Shc) and monoamine oxidase. Basic Res. Cardiol. 104 (2): 131-139. doi: 10.1007/s00395-009-0008-4.

Duicu, O.M., Lighezan, R., Sturza, A., Ceausu, R.A., Borza, C., and Vaduva, A. 2015. Monoamine Oxidases as Potential Contributors to Oxidative Stress in Diabetes: Time for a Study in Patients Undergoing Heart Surgery. Biomed. Res. Int. 515437. doi: $10.1155 / 2015 / 515437$.

Ebadi, M., Brown-Borg, H., Ren, J., Sharma, S., Shavali, S., El ReFaey, H., and Carlson, E.C. 2006. Therapeutic efficacy of selegiline in neurodegenerative disorders and neurological diseases. Current drug targets 7(11): 1513-1529.

Finberg, J.P., and Gillman, K. 2011. Selective inhibitors of monoamine oxidase type B and the "cheese effect". Int. Rev. Neurobiol. 100: 169-190. doi: 10.1016/b978-0-12-386467$3.00009-1$. 
Fridovich, I. 1995. Superoxide radical and superoxide dismutases. Annu. Rev. Biochem. 64: 97-112. doi: 10.1146/annurev.bi.64.070195.000525.

Griffith, G.C. 1960. Amine oxidase inhibitors; their current place in the therapy of cardiovascular diseases. Circulation, 22: 1156-1165.

Kaludercic, N., Carpi, A., Menabo, R., Di Lisa, F., and Paolocci, N. 2011. Monoamine oxidases (MAO) in the pathogenesis of heart failure and ischemia/reperfusion injury. Biochim. Biophys. Acta, 1813(7): 1323-1332. doi: 10.1016/j.bbamcr.2010.09.010.

Kaludercic, N., Carpi, A., Nagayama, T., Sivakumaran, V., Zhu, G., Lai, E.W., Bedja, D., De Mario, A., Chen, K., Gabrielson, K.L., Lindsey, M.L., Pacak, K., Takimoto, E., Shih, J.C., Kass, D.A., Di Lisa, F., and Paolocci, N. 2014. Monoamine oxidase B prompts mitochondrial and cardiac dysfunction in pressure overloaded hearts. Antioxid. Redox Signal. 20(2): 267280. doi: 10.1089/ars.2012.4616.

Kaludercic, N., Takimoto, E., Nagayama, T., Feng, N., Lai, E.W., Bedja, D., Chen, K., Gabrielson, K.L., Blakely, R.D., Shih, J.C., Pacak, K., Kass, D.A., Di Lisa, F., and Paolocci, N. 2010. Monoamine oxidase A-mediated enhanced catabolism of norepinephrine contributes to adverse remodeling and pump failure in hearts with pressure overload. Circ. Res. 106(1): 193-202. doi: 10.1161/circresaha.109.198366.

Kushelevskii, B.P., and Kokosov, A.N. 1967. Monoaminoxidase inhibitors in the treatment of angina pectoris. Sovetskaia meditsina, 30(4): 24-29.

Raasch, W., Bartels, T., Gieselberg, A., Dendorfer, A., and Dominiak, P. 2002. Angiotensin Iconverting enzyme inhibition increases cardiac catecholamine content and reduces monoamine oxidase activity via an angiotensin type 1 receptor-mediated mechanism. J. Pharmacol. Exp. Therapeut. 300(2): 428-434. 
Rosendorff, C., Lackland, D.T., Allison, M., Aronow, W.S., Black, H.R., Blumenthal, R.S., Cannon, C.P., de Lemos, J.A., Elliott, W.J., Findeiss, L., Gersh, B.J., Gore, J.M., Levy, D., Long, J.B., O'Connor, C.M., O'Gara, P.T., Ogedegbe, G., Oparil, S., and White, W.B. 2015. Treatment of hypertension in patients with coronary artery disease: a scientific statement from the American Heart Association, American College of Cardiology, and American Society of Hypertension. Hypertension, 65(6): 1372-1407. doi: 10.1161/hyp.0000000000000018.

Simon, P. 1970. Pharmacologic characteristics of monoamine oxidase inhibitors: problems related to their use in the treatment of angina. Therapie, 25(2): 343-348.

St-Pierre, J., Buckingham, J.A., Roebuck, S.J., and Brand, M.D. 2002. Topology of superoxide production from different sites in the mitochondrial electron transport chain. J. Biol. Chem. 277(47): 44784-44790. doi: 10.1074/jbc.M207217200.

Sturza, A., Duicu, O.M., Vaduva, A., Danila, M.D., Noveanu, L., Varro, A., and Muntean, D.M. 2015. Monoamine oxidases are novel sources of cardiovascular oxidative stress in experimental diabetes. Can. J. Physiol. Pharmacol. 93(7): 555-561. doi: 10.1139/cjpp-20140544 .

Sturza, A., Leisegang, M.S., Babelova, A., Schroder, K., Benkhoff, S., Loot, A.E., Fleming, I., Schulz, R., Muntean, D.M., and Brandes, R.P. 2013. Monoamine oxidases are mediators of endothelial dysfunction in the mouse aorta. Hypertension, 62(1): 140-146. doi: 10.1161/hypertensionaha.113.01314.

Sturza, A., Noveanu, L., Duicu, O., and Muntean, D.M. 2014. Monoamine oxidase inhibition corrects endothelial dysfunction in experimental diabetes. Cardiovasc. Res. 103 (Suppl. 1): S30-S30. doi: 10.1093/cvr/cvu082.108.

Thomas, T. 2000. Monoamine oxidase-B inhibitors in the treatment of Alzheimer's disease. Neurobiology of aging, 21(2): 343-348. 
Thomas, T., McLendon, C., and Thomas, G. 1998. L-deprenyl: nitric oxide production and dilation of cerebral blood vessels. Neuroreport, 9(11): 2595-2600.

Villeneuve, C., Guilbeau-Frugier, C., Sicard, P., Lairez, O., Ordener, C., Duparc, T., De Paulis, D., Couderc, B., Spreux-Varoquaux, O., Tortosa, F., Garnier, A., Knauf, C., Valet, P., Borchi, E., Nediani, C., Gharib, A., Ovize, M., Delisle, M.B., Parini, A., and Mialet-Perez, J. 2013. p53-PGC-1alpha pathway mediates oxidative mitochondrial damage and cardiomyocyte necrosis induced by monoamine oxidase-A upregulation: role in chronic left ventricular dysfunction in mice. Antioxid. Redox Signal. 18(1): 5-18. doi: 10.1089/ars.2011.4373. 
Table 1. Characteristics of the study groups.

\begin{tabular}{|c|c|c|}
\hline STUDY GROUPS & CHD $(n=24)$ & CHD-DM $(\mathrm{n}=22)$ \\
\hline \multicolumn{3}{|l|}{ Demographics } \\
\hline Age & $62 \pm 9$ & $60 \pm 7$ \\
\hline Sex, M/F (male/female) & $87.5 \% / 12.5 \%$ & $63.6 \% / 36.4 \%$ \\
\hline \multicolumn{3}{|l|}{ Clinical characteristics } \\
\hline Body Mass Index & $26.85 \pm 4.6$ & $30.8 \pm 2.7^{*}$ \\
\hline Cholesterol (mg/dL) & $154 \pm 33.6$ & $151 \pm 21$ \\
\hline Fasting plasma glucose (mg/dL) & $105 \pm 16.4$ & $192 \pm 65.3^{*}$ \\
\hline Left Ventricular Ejection Fraction & $48.4 \pm 8.5 \%$ & $48.5 \pm 7.3 \%$ \\
\hline Hypertension & $100 \%$ & $100 \%$ \\
\hline Atrial fibrillation & $4.2 \%$ & $0 \%$ \\
\hline \multicolumn{3}{|l|}{ Preoperative medication } \\
\hline Aspirin & $100 \%$ & $100 \%$ \\
\hline$\beta$-Blockers & $100 \%$ & $100 \%$ \\
\hline Anticoagulants & $100 \%$ & $100 \%$ \\
\hline Statins & $100 \%$ & $100 \%$ \\
\hline Nitrates & $72 \%$ & $73.3 \%$ \\
\hline Calcium channel blockers & $20 \%$ & $33.3 \%$ \\
\hline \multicolumn{3}{|l|}{ Angiotensin-converting-enzyme } \\
\hline inhibitors & $100 \%$ & $100 \%$ \\
\hline Diuretics & $100 \%$ & $100 \%$ \\
\hline Insulin & $0 \%$ & $20 \%$ \\
\hline Oral antidiabetics & $0 \%$ & $80 \%$ \\
\hline Antibiotherapy & $100 \%$ & $100 \%$ \\
\hline
\end{tabular}


Data are means \pm S.E.M. ${ }^{*} p<0.05:$ CHD vs. CHD-DM. 


\section{Figure captions}

Figure 1. The effect of MAO inhibitors on vasomotor function in human mammary arteries. A and B. Phenylephrine induced contractions (A) and acetylcholine-induced relaxation (B) in mammary artery segments isolated from patients with coronary heart disease without diabetes (CHD) in the presence vs. absence of irreversible MAO-A inhibitor clorgyline (CHD+CLORG, $10 \mu \mathrm{mol} / \mathrm{L})$, reversible MAO-A inhibitor moclobemide (CHD+MOCLOB, $10 \mu \mathrm{mol} / \mathrm{L})$ and irreversible MAO-B inhibitor selegiline (CHD+SELEG, $10 \mu \mathrm{mol} / \mathrm{L}) . \mathrm{n}=10,{ }^{*, \#}{ }^{\&}<0.05 \mathrm{CHD}$ with $v s$. without the named inhibitor. $\mathbf{C}$ and $\mathbf{D}$. Phenylephrine induced contractions (C) and acetylcholine-induced relaxation (D) in mammary artery segments isolated from patients with coronary artery disease with diabetes (CHD-DM) in the presence or absence of irreversible MAO-A inhibitor clorgyline (CHD$\mathrm{DM}+\mathrm{CLORG}, \quad 10 \mu \mathrm{mol} / \mathrm{L}), \quad$ reversible MAO-A inhibitor moclobemide (CHD$\mathrm{DM}+\mathrm{MOCLOB}, \quad 10 \mu \mathrm{mol} / \mathrm{L}$ ) and irreversible MAO-B inhibitor selegiline (CHD$\mathrm{DM}+\mathrm{SELEG}, 10 \mu \mathrm{mol} / \mathrm{L}) . \mathrm{n}=10,{ }^{* \#, \&} p<0.05$ CHD-DM with $v s$. without the named inhibitor.

Figure 2. Segments of mammary artery from non-diabetic and diabetic CHD patients stained with Hematoxylin-Eosin. A) CHD patients, B) CHD-DM patients.

Figure 3. MAO expression in human mammary arteries segments from non-diabetic CHD patients. Immune-histology for MAO-A (A) and MAO-B (B):(a\&d) entire arterial wall, (b\&e) endothelial layer and muscular media, (c\&f) adventitia with fibroblasts, nerves and vasa-vasorum. $E C=$ endothelial cell, $F B=$ fibroblast, $I E L=$ internal elastic lamina 
Figure 4. MAO expression in human mammary arteries segments from diabetic CHD patients. Immune-histology for MAO-A (A) and MAO-B (B): (a\&d) entire arterial wall, (b\&e) endothelial layer and muscular media, (c\&f) adventitia with fibroblasts, nerves and vasa-vasorum. $E C=$ endothelial cell, $F B=$ fibroblast, $I E L=$ internal elastic lamina

Figure 5. The effect of MAO inhibitors on vasomotor function in endothelium denuded human mammary arteries. A and B. Phenylephrine induced contractions (A) and acetylcholine-induced relaxation (B) in endothelium denuded mammary artery segments isolated from patients with coronary artery disease without diabetes - $\mathrm{CHD}(\mathrm{e}-)$ in the presence or absence of irreversible MAO-A inhibitor clorgyline-CHD(e-)+CLORG, $10 \mu \mathrm{mol} / \mathrm{L}$, reversible MAO-A inhibitor moclobemide - $\mathrm{CHD}(\mathrm{e}-)+\mathrm{MOCLOB}, 10 \mu \mathrm{mol} / \mathrm{L})$ and irreversible MAO-B inhibitor selegiline - CHD(e-)+SELEG, $10 \mu \mathrm{mol} / \mathrm{L} . \mathrm{n}=10,{ }^{*, \#, \&} p<0.05$ CHD(e-)with vs. without the named inhibitor. C and D. Phenylephrine induced contractions (C) and acetylcholine-induced relaxation (D) in endothelium denuded mammary artery segments isolated from patients with coronary artery disease with diabetes - CHD-DM(e-) in the presence or absence of irreversible MAO-A inhibitor clorgyline - CHD-DM(e-)+CLORG, 10 $\mu \mathrm{mol} / \mathrm{L}$, reversible MAO-A inhibitor moclobemide - CHD-DM(e-)+MOCLOB, $10 \mu \mathrm{mol} / \mathrm{L}$, and irreversible MAO-B inhibitor selegiline - CHD-DM(e-)+SELEG, $10 \mu \mathrm{mol} / \mathrm{L} . \mathrm{n}=10$, *,\#\& $p<0.05$ CHD-DM(e-) with $v s$. without the named inhibitor. 

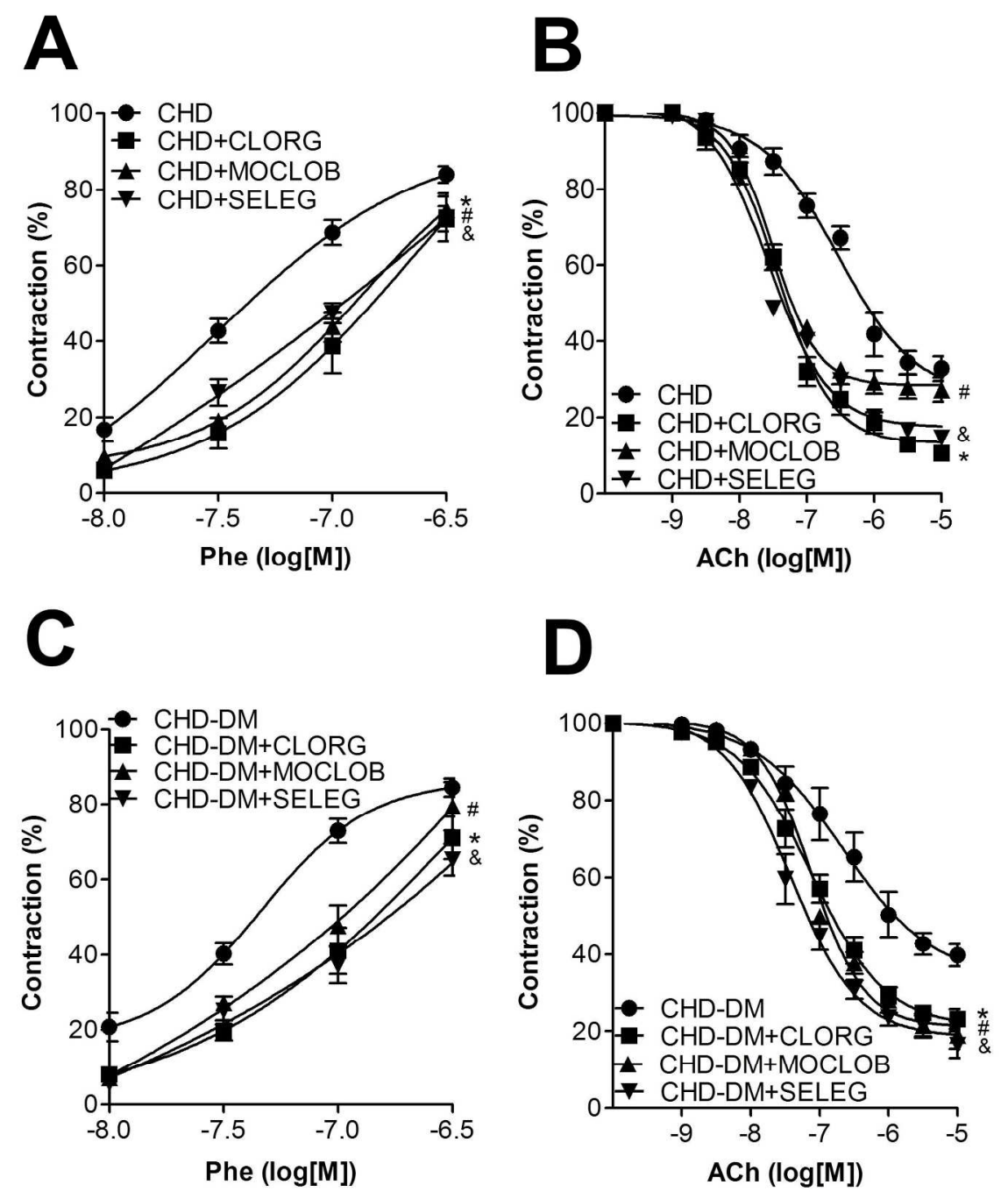

Figure 1. The effect of MAO inhibitors on vasomotor function in human mammary arteries. A and $B$. Phenylephrine induced contractions (A) and acetylcholine-induced relaxation (B) in mammary artery segments isolated from patients with coronary heart disease without diabetes (CHD) in the presence vs. absence of irreversible MAO-A inhibitor clorgyline (CHD+CLORG, $10 \mu \mathrm{mol} / \mathrm{L})$, reversible MAO-A inhibitor moclobemide (CHD+MOCLOB, $10 \mu \mathrm{mol} / \mathrm{L})$ and irreversible MAO-B inhibitor selegiline (CHD+SELEG, 10 $\mu \mathrm{mol} / \mathrm{L}) . \mathrm{n}=10, *, \#, \& \mathrm{p}<0.05 \mathrm{CHD}$ with vs. without the named inhibitor. C and D. Phenylephrine induced contractions (C) and acetylcholine-induced relaxation (D) in mammary artery segments isolated from patients with coronary artery disease with diabetes (CHD-DM) in the presence or absence of irreversible MAO-A inhibitor clorgyline (CHD-DM+CLORG, $10 \mu \mathrm{mol} / \mathrm{L}$ ), reversible MAO-A inhibitor moclobemide (CHD$\mathrm{DM}+\mathrm{MOCLOB}, 10 \mu \mathrm{mol} / \mathrm{L}$ ) and irreversible MAO-B inhibitor selegiline (CHD-DM+SELEG, $10 \mu \mathrm{mol} / \mathrm{L}) . \mathrm{n}=10$, $*, \#, \& \mathrm{p}<0.05 \mathrm{CHD}-\mathrm{DM}$ with vs. without the named inhibitor. $254 \times 338 \mathrm{~mm}(300 \times 300 \mathrm{DPI})$ 

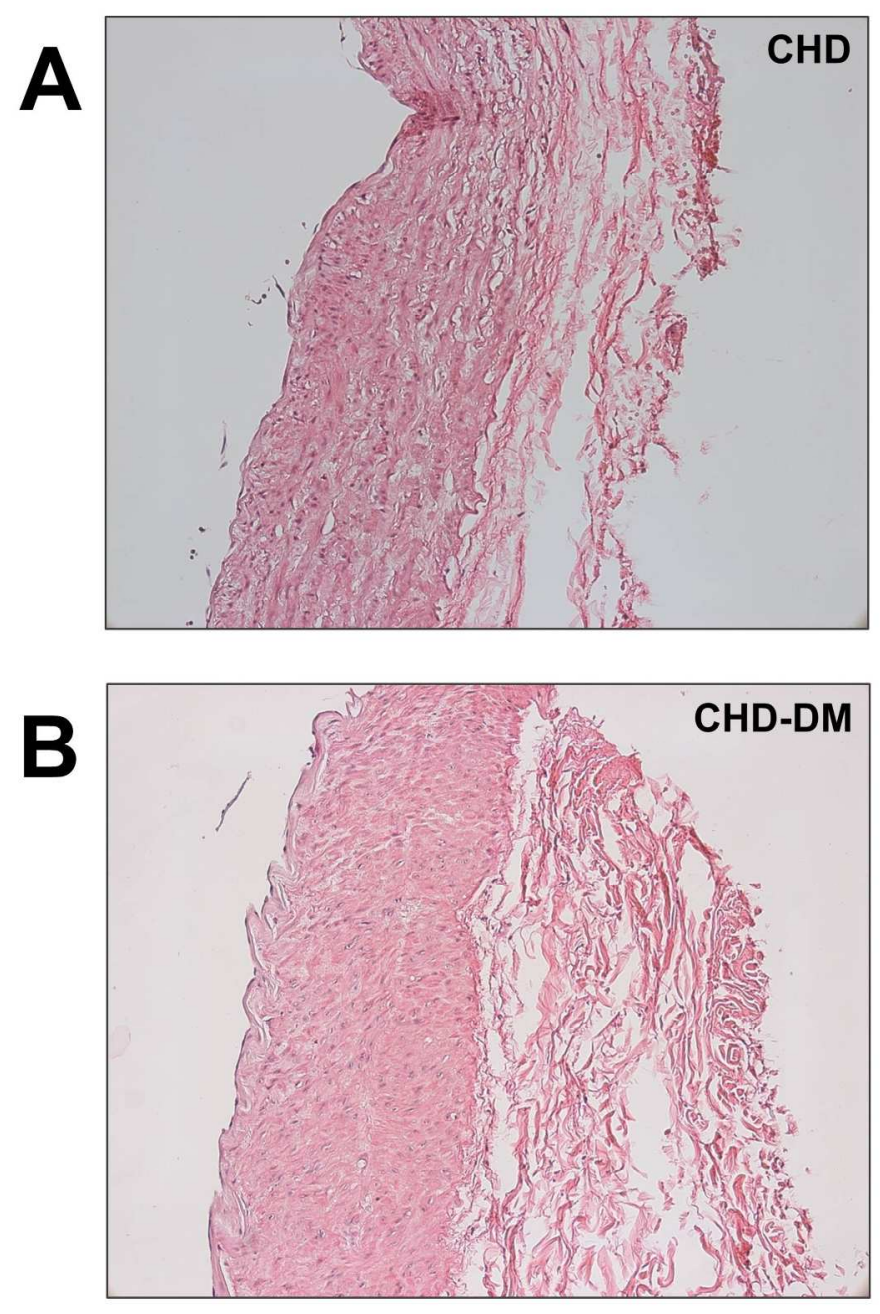

Figure 2. Segments of mammary artery from non-diabetic and diabetic CHD patients stained with Hematoxylin-Eosin. A) CHD patients, B) CHD-DM patients. $254 \times 338 \mathrm{~mm}(300 \times 300 \mathrm{DPI})$ 

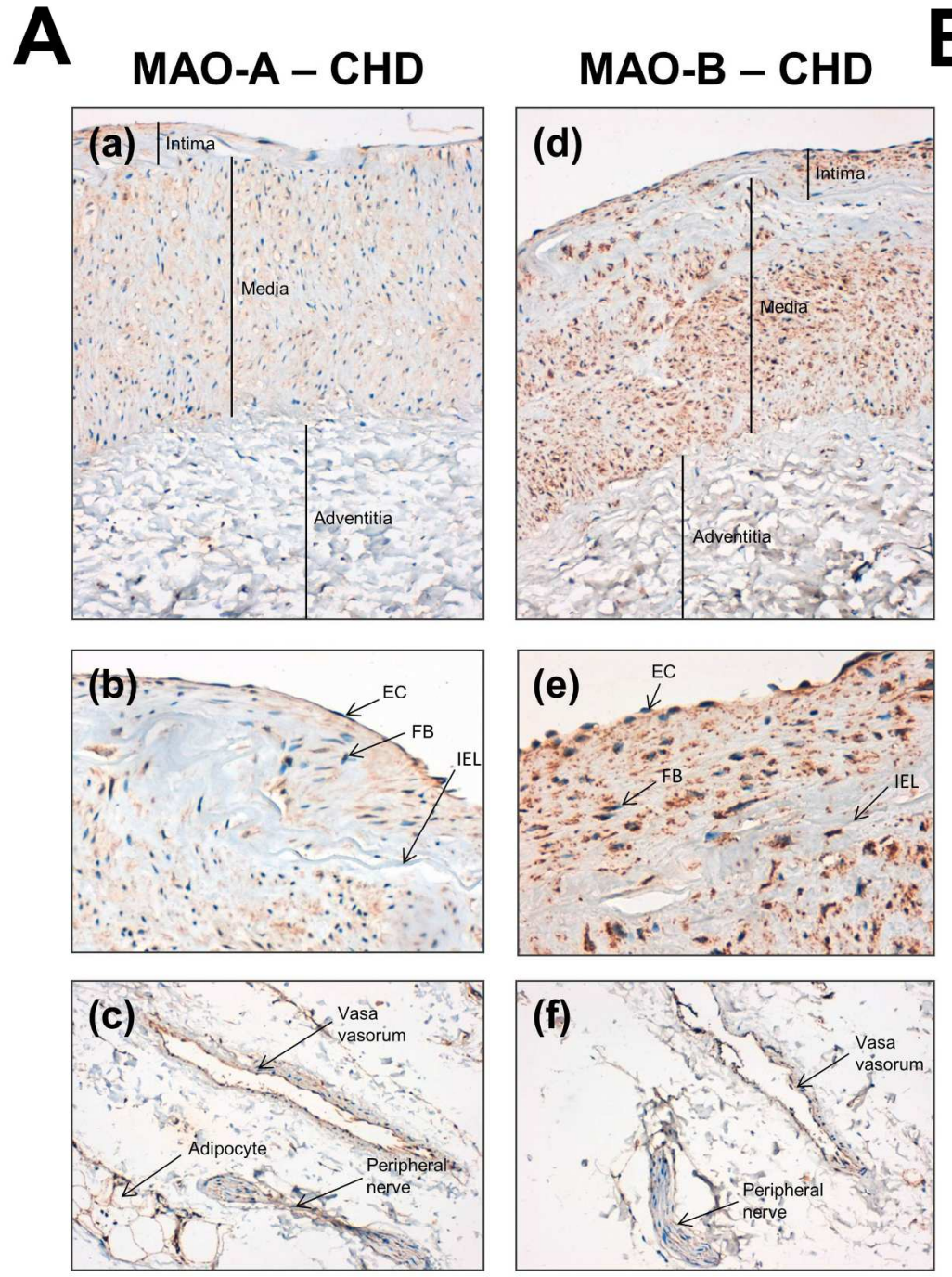

Figure 3. MAO expression in human mammary arteries segments from non-diabetic CHD patients. Immunehistology for MAO-A (A) and MAO-B (B):(a\&d) entire arterial wall, (b\&e) endothelial layer and muscular media, (c\&f) adventitia with fibroblasts, nerves and vasa-vasorum. EC= endothelial cell, FB= fibroblast, IEL $=$ internal elastic lamina

$254 \times 338 \mathrm{~mm}(300 \times 300$ DPI $)$ 
A

\section{MAO-A - CHD-DM}
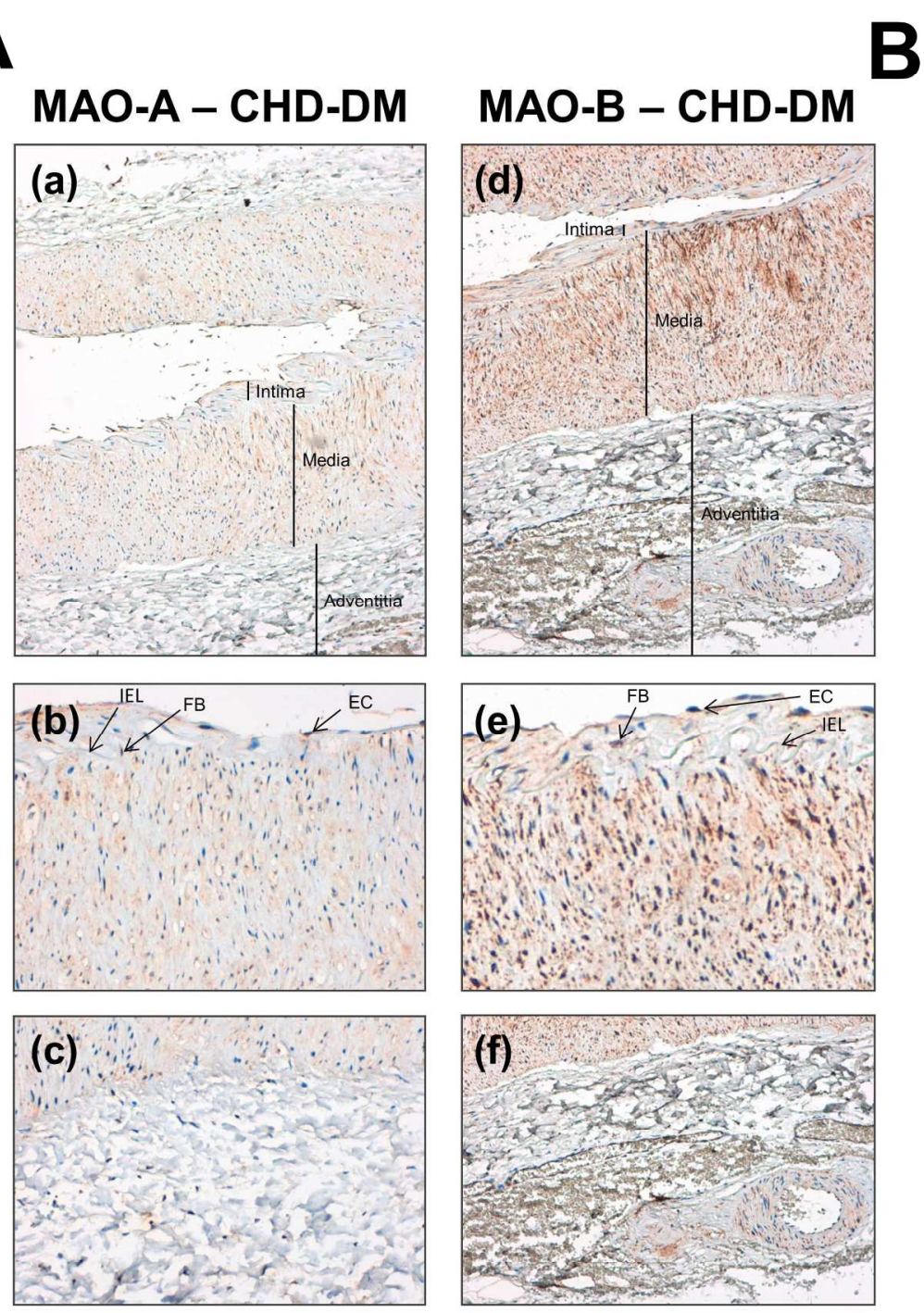

Figure 4. MAO expression in human mammary arteries segments from diabetic CHD patients. Immunehistology for MAO-A (A) and MAO-B (B): (a\&d) entire arterial wall, (b\&e) endothelial layer and muscular media, (c\&f) adventitia with fibroblasts, nerves and vasa vasorum. $E C=$ endothelial cell, $F B=$ fibroblast, $I E L=$ internal elastic lamina $254 \times 338 \mathrm{~mm}(300 \times 300 \mathrm{DPI})$ 

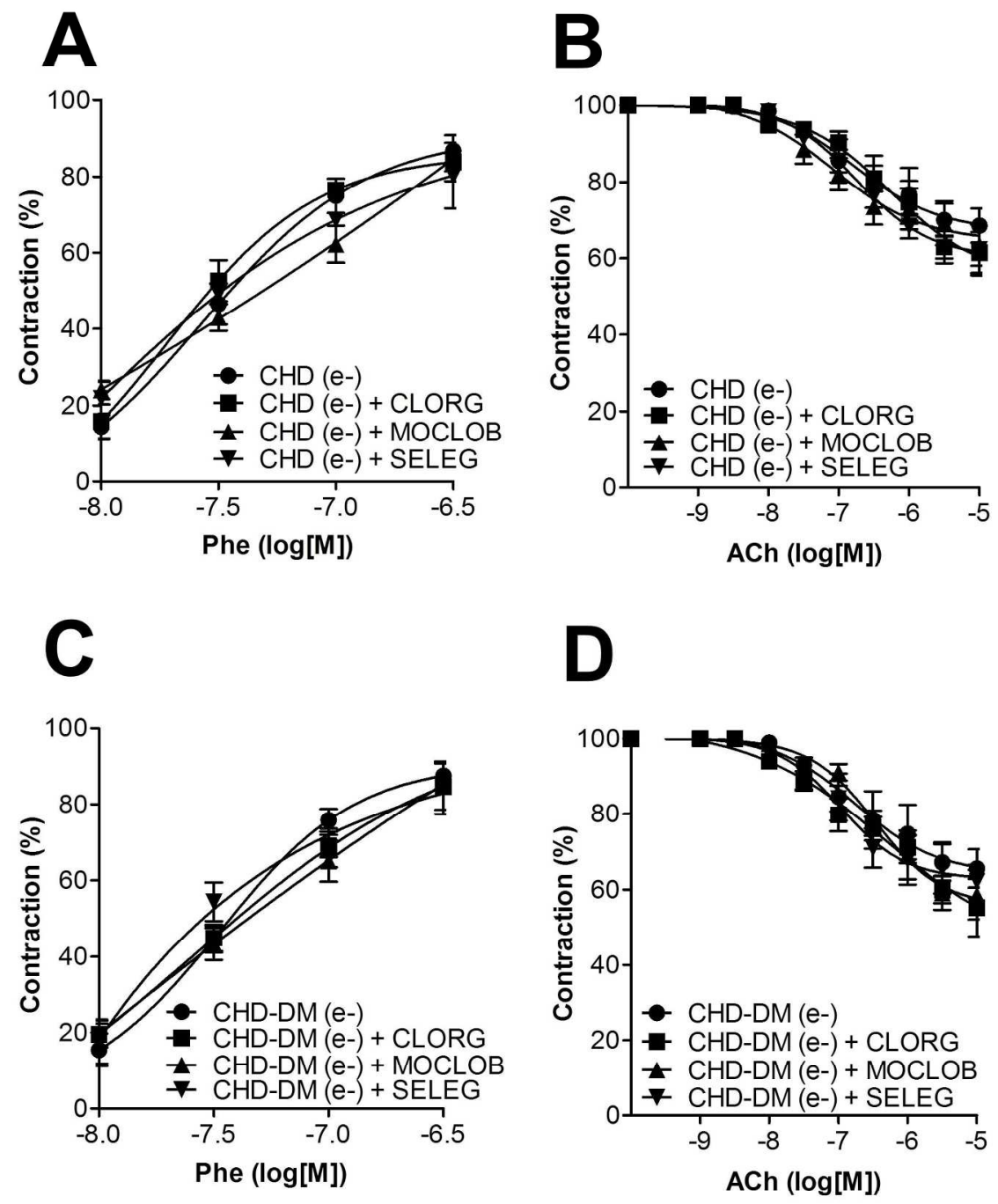

Figure 5. The effect of MAO inhibitors on vasomotor function in endothelium denuded human mammary arteries. A and B. Phenylephrine induced contractions (A) and acetylcholine-induced relaxation (B) in endothelium denuded mammary artery segments isolated from patients with coronary artery disease without diabetes - $\mathrm{CHD}(\mathrm{e}-)$ in the presence or absence of irreversible MAO-A inhibitor clorgyline-CHD(e)+CLORG, $10 \mu \mathrm{mol} / \mathrm{L}$, reversible MAO-A inhibitor moclobemide - CHD(e-)+MOCLOB, $10 \mu \mathrm{mol} / \mathrm{L})$ and irreversible MAO-B inhibitor selegiline - CHD(e-)+SELEG, $10 \mu \mathrm{mol} / \mathrm{L} . \mathrm{n}=10, *, \#, \& p<0.05 \mathrm{CHD}(\mathrm{e}-)$ with vs. without the named inhibitor. C and D. Phenylephrine induced contractions (C) and acetylcholine-induced relaxation (D) in endothelium denuded mammary artery segments isolated from patients with coronary artery disease with diabetes - CHD-DM(e-) in the presence or absence of irreversible MAO-A inhibitor clorgyline - CHD-DM(e-)+CLORG, $10 \mu \mathrm{mol} / \mathrm{L}$, reversible MAO-A inhibitor moclobemide - CHD-DM(e)+MOCLOB, $10 \mu \mathrm{mol} / \mathrm{L}$, and irreversible MAO-B inhibitor selegiline - CHD-DM(e-)+SELEG, $10 \mu \mathrm{mol} / \mathrm{L} . \mathrm{n}=10$, *,\#,\&p<0.05 CHD-DM(e-) with vs. without the named inhibitor. 
